\title{
Multi-parametric arterial spin labelling and diffusion-weighted magnetic resonance imaging in differentiation of grade II and grade III gliomas
}

\author{
Ahmed Abdel Khalek Abdel Razek ${ }^{1 A, C, D, E, F}$, Lamiaa Galal El-Serougy ${ }^{10}$, Mohamed A. Abdelsalam ${ }^{20, E}$, \\ Gada Mohamed Gaballa ${ }^{1 B, C}$, Mona Mohamed Talaat ${ }^{3 C, D}$ \\ 'Department of Diagnostic Radiology, Mansoura Faculty of Medicine, Mansoura, Egypt \\ 2Department of Neurology, Mansoura Faculty of Medicine, Mansoura, Egypt \\ ${ }^{3}$ Department of Diagnostic Radiology, Kafr Elsheak Faculty of Medicine, Kafr Elsheikh, Egypt
}

\section{Abstract}

Purpose: To assess arterial spin labelling (ASL) perfusion and diffusion MR imaging (DWI) in the differentiation of grade II from grade III gliomas.

Material and methods: A prospective cohort study was done on 36 patients (20 male and 16 female) with diffuse gliomas, who underwent ASL and DWI. Diffuse gliomas were classified into grade II and grade III. Calculation of tumoural blood flow (TBF) and apparent diffusion coefficient (ADC) of the tumoral and peritumoural regions was made. The ROC curve was drawn to differentiate grade II from grade III gliomas.

Results: There was a significant difference in TBF of tumoural and peritumoural regions of grade II and III gliomas ( $p=0.02$ and $p=0.001$, respectively). Selection of 26.1 and $14.8 \mathrm{ml} / 100 \mathrm{~g} / \mathrm{min}$ as the cut-off for TBF of tumoural and peritumoural regions differentiated between both groups with area under curve (AUC) of 0.69 and 0.957, and accuracy of $77.8 \%$ and $88.9 \%$, respectively. There was small but significant difference in the ADC of tumoural and peritumoural regions between grade II and III gliomas ( $p=0.02$ for both). The selection of 1.06 and $1.36 \times 10^{-3} \mathrm{~mm}^{2} / \mathrm{s}$ as the cut-off of ADC of tumoural and peritumoural regions was made, to differentiate grade II from III with AUC of 0.701 and 0.748 , and accuracy of $80.6 \%$ and $80.6 \%$, respectively. Combined TBF and ADC of tumoural regions revealed an AUC of 0.808 and accuracy of $72.7 \%$. Combined TBF and ADC for peritumoural regions revealed an AUC of 0.96 and accuracy of $94.4 \%$.

Conclusion: TBF and ADC of tumoural and peritumoural regions are accurate non-invasive methods of differentiation of grade II from grade III gliomas.

Key words: arterial spin labelling, diffusion weighted imaging, glioma, grading.

\section{Introduction}

Differentiation between World Health Organisation (WHO) grade II and III gliomas is of upmost importance for selecting and developing appropriate treatment, detecting early treatment failure, and identifying accurate and clinically relevant biological endpoints for high-risk but potentially highly rewarding tumoural specific therapies tailored to the unique biology of an individual brain tumour [1-4]. Another benefit of imaging is the possibility of identifying the most malignant areas within a tumour in order to minimise the risk of biopsy sampling errors. Histopathology is the gold standard for grading of gliomas, but it carries sampling error due to the limited number of biopsy samples [5-8]. Conventional pre- and post-contrast MR imaging [9] and advanced magnetic

Correspondence address:

Ahmed Abdel Khalek Abdel Razek, Department of Diagnostic Radiology, Mansoura Faculty of Medicine, Mansoura University, Mansoura, Egypt, 13551,

phone: 00201061948567, e-mail: arazek@mans.edu.eg

Authors' contribution:

A Study design · B Data collection · C Statistical analysis · D Data interpretation - E Manuscript preparation · F Literature search · G Funds collection 
resonance $(\mathrm{MR})$ imaging techniques such as dynamic contrast enhancement and dynamic susceptibility contrast MR imaging [10-13], diffusion-weighted MR imaging $[14,15]$, or combined MR pulse sequences $[16,17]$ are used to differentiate grade II from grade III gliomas, but their results are overlapping [10-17].

Arterial spin labelling (ASL) is a non-invasive, perfusion-weighted MR imaging technique that calculates cerebral perfusion maps without administration of intravenous contrast agent [18-22]. The main principle of ASL is to use water protons of the arterial blood itself as a contrast agent to measure perfusion. The magnetisation of these inflowing protons is inverted in a region proximal to the scanned area by an external radiofrequency pulse [21-28]. Diffusion-weighted MR imaging has shown potential value in differentiating between glioma grades and in predicting the outcome of gliomas. It was reported that the histopathological grade of gliomas is inversely correlated with the apparent diffusion coefficient (ADC) value [29-32]. Few studies have discussed the role of ASL [21-24] and diffusion-weighted MR imaging [29-32] in the grading of gliomas. The novelty and uniqueness of this study is assessing combined tumour blood flow (TBF) and ADC of tumoural and peritumoural regions in the differentiation of grade II and grade III gliomas.

Its purpose is to assess ASL and DWI in the differentiation of grade II and grade III gliomas.

\section{Material and methods}

\section{Patients}

This prospective study was approved by the Local Ethics Committee, and informed consent was obtained from each patient prior to the examination. The study included 39 patients provisionally diagnosed to have untreated diffuse gliomas based on conventional MR imaging. We excluded three patients from the study because two patients had metastasis and one patient had lymphoma. Finally, the study included 36 patients (20 male and 16 female). Their age ranged from 52-72 years (mean age, 62 years). The final diagnosis was based on histopathological examinations. Gliomas were classified according to world health organisation (WHO) classification into WHO grade II for 19 patients and WHO grade III for 17 patients.

\section{Routine magnetic resonance imaging}

The MR imaging of the brain was performed using a 1.5 Tesla MRI scanner (Ingenia, Philips) using a Stream Head Neck 20 channel coil. T1-weighted (TR/TE $=600 / 25 \mathrm{~ms})$, T2-weighted $(\mathrm{TR} / \mathrm{TE}=6000 / 90 \mathrm{~ms})$, and FLAIR $(\mathrm{TR} /$ $\mathrm{TE} / \mathrm{TI}=10000 / 115 / 2700 \mathrm{~ms}$ ) sequences were obtained. The scanning parameters were matrix $=80 \times 80$, field of view $(\mathrm{FOV})=250 \times 170 \mathrm{~mm}^{2}$, and slice thickness $=5 \mathrm{~mm}$. Post-contrast T1-weighted images were obtained after intra- venous administration of gadoterate meglumine, $0.5 \mathrm{ml} / \mathrm{kg}$ $(0.1 \mathrm{mmol} / \mathrm{kg})$ body weight, with maximum dose of $10 \mathrm{ml}$ using a 20-22 G venous cannula with flow rate of $2 \mathrm{ml} / \mathrm{s}$.

\section{Arterial spin labelling imaging}

ASL perfusion imaging was performed with pseudo-continuous labelling. Multiple time points were acquired after the label pulse. Fast spin echo single-shot EPI parallel imaging sequence SENSE was used with reduction of P 2.3 to reduce echo train length susceptibility-related distortions. The following parameters were used: slice thickness $=8 \mathrm{~mm}$, inter-slice gap $=1.5 \mathrm{~mm}, \mathrm{FOV}=240 \times 240 \mathrm{~mm}$, data matrix $=68 \times 68$, voxel size $=3.6 \times 3.5 \times 3 \mathrm{~mm}, \mathrm{TR} / \mathrm{TE}=250 /$ $20 \mathrm{~ms}$, slice number $=6$, scan duration $=4 \mathrm{~min}$, and postlabelling delay time $=1.5 \mathrm{~s}$.

\section{Diffusion-weighted imaging}

Imaging was done in the axial plane using multiple $b$ values ( $b$ values of 0 and $1000 \mathrm{~s} / \mathrm{mm}^{2}$ ). It was performed before administration of contrast medium by using a single-shot, spin-echo, echo-planar imaging sequence with the following parameters: slice thickness $=8 \mathrm{~mm}$, inter-slice gap $=1.5$, FOV $=240 \times 240 \mathrm{~mm}$, voxel size $=1.51 \times 2.17 \times 5 \mathrm{~mm}$, $\mathrm{TR} / \mathrm{TE}=3004 / 88 \mathrm{~ms}$, and scan duration $=1 \mathrm{~min}$.

\section{Post processing}

Image analysis was performed by one radiologist expert in neuroradiology with 10 years' experience, who was blinded to the histopathological results. The DICOM images were transferred to a workstation (extended MR Workspace 2.6.3.5, Philips medical systems Nederland BV) supplied by the vendor. The post processing of arterial spin-tagging data typically involves initial subtraction of alternating tag and control image pairs and motion correction. The regions of interest (ROIs) were placed within tumoural and peritumoural regions (within a $1 \mathrm{~cm}$ distance from the outer enhancing tumoural margin). The ROIs were drawn in an area with maximum signal enhancement on an ASL map and the lowest signal on ADC. Necrotic tissue and large vessels were avoided by comparison with T1Gd and T2-weighted images. The TBF was calculated as previously described [20].

\section{Statistical analysis}

Statistical analyses were carried out using Statistical Package for Social Sciences version 20 (SPSS, Chicago, IL). The data was normally distributed. Quantitative data were presented as mean \pm standard deviation (SD). Normally distributed data were compared between the two major groups using independent samples $t$ test and between other groups. Probability $(p)$ values $<0.05$ were considered statistically significant. The receiver operating characteris- 
tic (ROC) curves of different matrices of the tumoural and peritumoural regions were done with calculation of the area under the curve (AUC). The optimum cut-off values of different metrics of tumoural and peritumoural regions with the highest accuracy were selected to differentiate grade II from grade III gliomas were calculation of accuracy, sensitivity, and specificity. The multivariate linear regression analysis was done for variables that reached a $p$ value of 0.05 for the best combination of TBF and $\mathrm{ADC}$ metrics in the tumoural and peritumoural regions used to differentiate grade II from grade III gliomas.

\section{Results}

Based on histopathological results, the patients were divided into grade II gliomas (Figure 1$)(n=19)$ and grade III gliomas $(n=17)$. Table 1 shows the mean, standard deviation, minimum, maximum, TBF, and $\mathrm{ADC}$ of tumoural and peritumoural regions of grade II and grade III gliomas. Table 2 shows the ROC results of TBF and ADC of tumoural and peritumoral regions of grade II and grade III gliomas.

The mean TBF of tumoural and peritumoural regions of grade II glioma were $25.56 \pm 3.34$ and 13.54 $\pm 1.48 \mathrm{ml} / 100 \mathrm{~g} / \mathrm{min}$, and for grade III glioma they were $27.98 \pm 2.98$ and $16.54 \pm 1.06 \mathrm{ml} / 100 \mathrm{~g} / \mathrm{min}$, respectively, with a significant difference $(p=0.02$ and $p=0.001)$. Selection of 26.1 and $14.8 \mathrm{ml} / 100 \mathrm{~g} / \mathrm{min}$ as the cut-off values of TBF of the tumoural and peritumoural regions to differentiate between the two groups revealed AUC of 0.69 and 0.957 , accuracy of $77.8 \%$ and $88.9 \%$, sensitivity of $82.4 \%$ and $94.1 \%$, and specificity of $73.7 \%$ and $84.2 \%$, respectively (Figure 2).

The mean ADC of tumoural and peritumoural regions of grade II gliomas were $1.18 \pm 0.11$ and $1.52 \pm 0.21$ $\times 10^{-3} \mathrm{~mm}^{2} / \mathrm{s}$, and for grade III gliomas they were $1.05 \pm$ 0.22 and $1.37 \pm 0.16\left(10^{-3} \mathrm{~mm}^{2} / \mathrm{s}\right)$, respectively, with a significant difference for both regions $(p=0.02)$. Selection of 1.06 and $1.36 \times 10^{-3} \mathrm{~mm}^{2} / \mathrm{s}$ as cut-off values of $\mathrm{ADC}$ of the tumoural and peritumoural regions to differentiate between grade II and III gliomas revealed AUC of 0.701 and 0.748 , accuracy of $80.6 \%$ and $80.6 \%$, sensitivity of $70.6 \%, 82.6 \%$, and specificity of $89.5 \%$ and $78.9 \%$, respectively (Figure 3 ).

Combined TBF and ADC of the tumoural region used for differentiation of grade II from grade III gliomas revealed AUC of 0.808 , accuracy of $2.7 \%$, sensitivity of $70.6 \%$, and specificity of $73.7 \%$. Combined TBF and $\mathrm{ADC}$ of the peritumoural region used for differentiation of grade II from grade III gliomas revealed AUC of 0.96, accuracy of $94.4 \%$, sensitivity of $94.1 \%$, and specificity of 94.1\% (Figure 4).

\section{Discussion}

The main findings in this study are that there is significant difference in $\mathrm{TBF}$ of tumoural and peritumoural regions of grade II and grade III gliomas $(p=0.02$ and $p=0.001$, respectively). There is a significant difference in ADC of tumoural and peritumoral regions between grade II and grade III gloms $(p=0.02)$. Multi-parametric TBF and $\mathrm{ADC}$ of the tumoural and peritumoural regions increased the diagnostic performance of MR imaging in differentiation of grade II and grade III gliomas. This study is different from other multi-parameter studies because we used two advanced MR sequences without the application of contrast medium, which reflects the cellularity and vascularity of the gliomas.

In this study, there was slightly significant difference of TBF of the tumoural region differentiating grade II from grade III gliomas $(p=0.02)$, while the peritumoural region TBF measures revealed a highly significant difference between both groups $(p=0.001)$. Other studies done using ASL techniques distinguishing high- from low-grade gliomas based on tumoural region measurements revealed higher CBF in high-grade than in low-grade gliomas with highly significant difference $[20,22,24]$. Another study added that the use of ASL-normalised vascular intratumoural signal intensity values allows differentiation between low-grade and high-grade gliomas and thus may serve as a new, non-invasive marker for astrocytoma grading [23]. One study revealed that TBF by ASL enabled discrimination of astrocytomas with and without IDH mutation $(p=0.014)$ and trial for discrimination astrocytomas with $I D H$ mutation from oligodendrogliomas $(p=0.074)[21]$.

In this study, there was a lower ADC value of the tumoural and peritumoural regions of grade III gliomas compared to grade II, with a slightly significant difference of ADC values measured in the tumoural region differentiating grade II from grade III gliomas $(p=0.02)$. One study reported that the $\mathrm{ADC}$ value calculated from $\mathrm{ADC}$ maps obtained from high $b$ values is a better imaging biomarker in the grading of gliomas [29]. Other studies reported that lower ADC values are used to discriminate high- from low-grade gliomas [30,33]. Another study using DTI technique showed a significant difference in ADC measured at the tumoural region $(p=0.001)$, while measurements at the peritumoural region showed only a less significant difference $(p=0.042)$ [32].

In this study, multi-parametrics of TBF and ADC measured at tumoural and peritumoural regions of gliomas increased the diagnostic performance of grading of gliomas. The best combination for differentiation between grade II and III gliomas is the TBF of the peritumoural regions, showing an accuracy of $94.4 \%$ with AUC 0.96 . Another study reported that the combination of ASL and conventional MR imaging gave good performance, with a diagnostic accuracy of $81.40 \%$ [24].

The advantages of ASL are the absolute values of perfusion of tissue by blood, without injected contrast agent or ionising radiation [20-28]. The advantages of diffusion-weighted MR imaging are its non-invasive, 

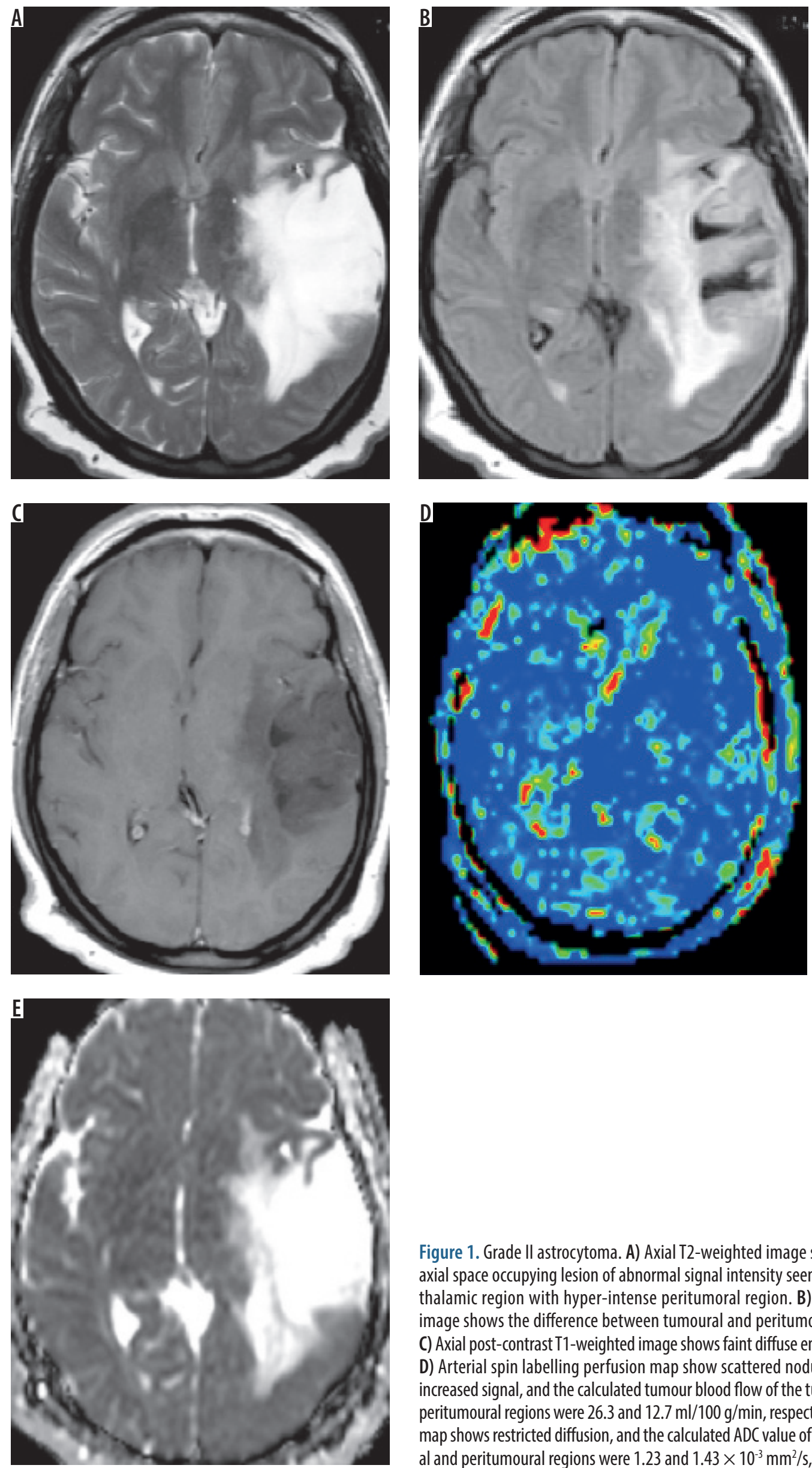

Figure 1. Grade II astrocytoma. A) Axial T2-weighted image shows intraaxial space occupying lesion of abnormal signal intensity seen in the right thalamic region with hyper-intense peritumoral region. B) Axial FLAIR image shows the difference between tumoural and peritumoural region. C) Axial post-contrast T1-weighted image shows faint diffuse enhancement. D) Arterial spin labelling perfusion map show scattered nodular areas of increased signal, and the calculated tumour blood flow of the tumoural and peritumoural regions were 26.3 and $12.7 \mathrm{ml} / 100 \mathrm{~g} / \mathrm{min}$, respectively. E) ADC map shows restricted diffusion, and the calculated $A D C$ value of the tumoural and peritumoural regions were 1.23 and $1.43 \times 10^{-3} \mathrm{~mm}^{2} / \mathrm{s}$, respectively 
Table 1. Mean, SD, minimum, maximum, $\mathrm{p}$ value of TBF and ADC of tumoural and peritumoural regions of grade II and III gliomas

\begin{tabular}{|l|c|c|c|}
\hline Parameter & $\begin{array}{c}\text { Grade II } \\
n=19\end{array}$ & $\begin{array}{c}\text { Grade III } \\
n=17\end{array}$ & $p$ value \\
\hline TBF peritumoural region & $13.54 \pm 1.48$ & $16.54 \pm 1.06$ & 0.001 \\
\hline TBF tumoural region & $25.56 \pm 3.34$ & $27.98 \pm 2.98$ & 0.02 \\
\hline ADC peritumoural region & $1.52 \pm 0.21$ & $1.37 \pm 0.16$ & 0.02 \\
\hline ADC tumoural region & $1.18 \pm 0.11$ & $1.05 \pm 0.22$ & 0.02 \\
\hline
\end{tabular}

TBF - tumour blood flow, ADC - apparent diffusion coefficient

Table 2. ROC of ASL, ADC, and combined parameters at tumoural and peritumoural regions of grade II and grade III gliomas with calculation of AUC, accuracy, specificity, and sensitivity

\begin{tabular}{|l|c|c|c|c|c|}
\hline Parameter & AUC & Cut off point & Sensitivity & Specificity & Accuracy \\
\hline TBF peritumoural region & 0.957 & 14.8 & 94.1 & 84.2 & 88.9 \\
\hline TBF tumoural region & 0.69 & 26.1 & 82.4 & 73.7 & 77.8 \\
\hline ADC peritumoural region & 0.748 & 1.36 & 82.4 & 78.9 & 80.6 \\
\hline ADC tumoural region & 0.701 & 1.06 & 70.6 & 89.5 & 80.6 \\
\hline Combined TBF \& ASL of peritumoural & 0.96 & & 94.1 & 94.7 & 94.4 \\
\hline Combined TBF \& ASL of tumoural & 0.808 & & 70.6 & 73.7 & 72.7 \\
\hline
\end{tabular}

TBF - tumoural blood flow, $A D C$ - apparent diffusion coefficient, $A S L$ - arterial spin labelling

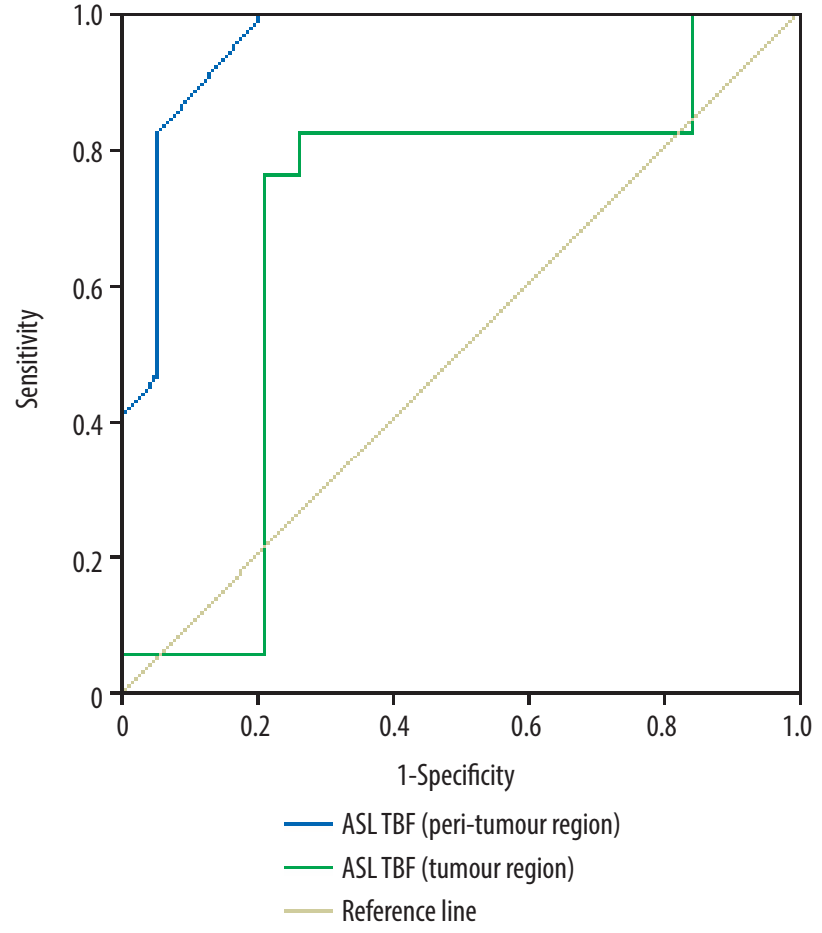

Figure 2. ROC curve of tumour blood flow (TBF). Selection of $26.1 \mathrm{ml} /$ $100 \mathrm{~g} / \mathrm{min}$ as a cut-off point of TBF at the tumoural region to differentiate grade II from grade III gliomas revealed AUC of 0.69 and an accuracy of $77.8 \%$. Selection of $14.8 \mathrm{ml} / 100 \mathrm{~g} / \mathrm{min}$ as a cut-off point of TBF at the peritumoral region to differentiate grade II from grade III gliomas revealed AUC of 0.957 and an accuracy of $88.9 \%$

rapid nature, which gives information about cellularity of the tumours, and without injection of contrast agent [34-39].

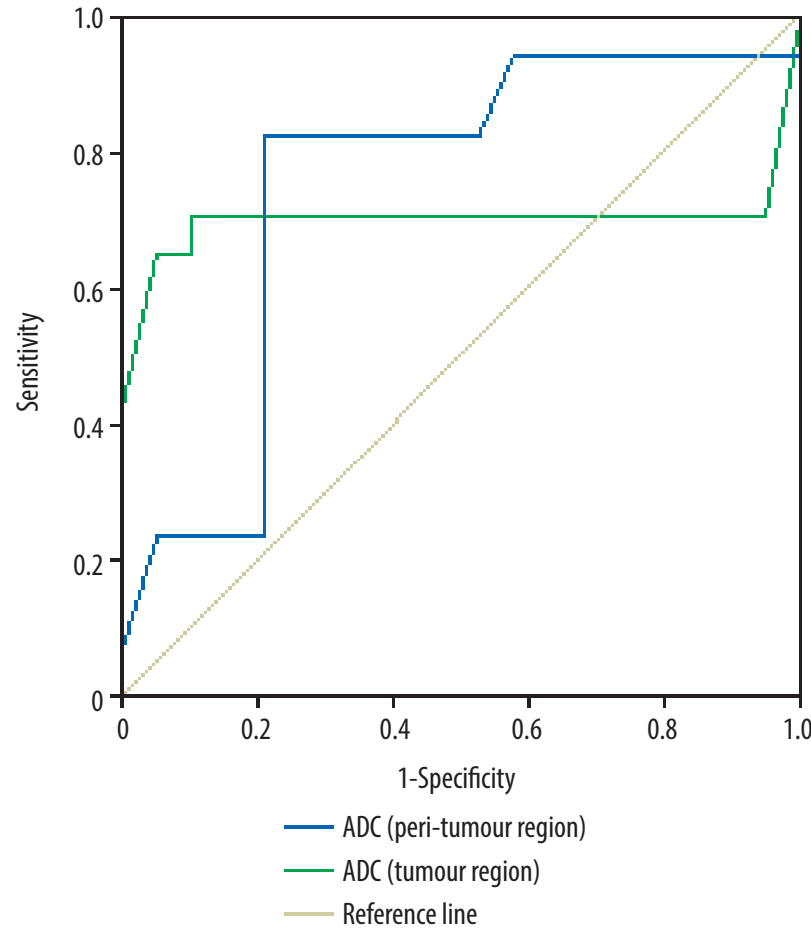

Figure 3. ROC curve of apparent diffusion coefficient (ADC). Selection of 1.06 $\times 10^{-3} \mathrm{~mm}^{2} / \mathrm{s}$ as a cut-off point of $A D C$ of tumoural region to differentiate grade II from grade III gliomas revealed AUC of 0.701 and accuracy of $80.6 \%$. Selection of $1.36 \times 10^{-3} \mathrm{~mm}^{2} / \mathrm{s}$ as a cut-off point of ADC of peritumoural region to differentiate grade II from grade III gliomas revealed AUC of 0.748 and an accuracy of $80.6 \%$

There are some limitations to this study. First, the small number of patients limits the statistical results. We recommended further studies with larger numbers of patients. 

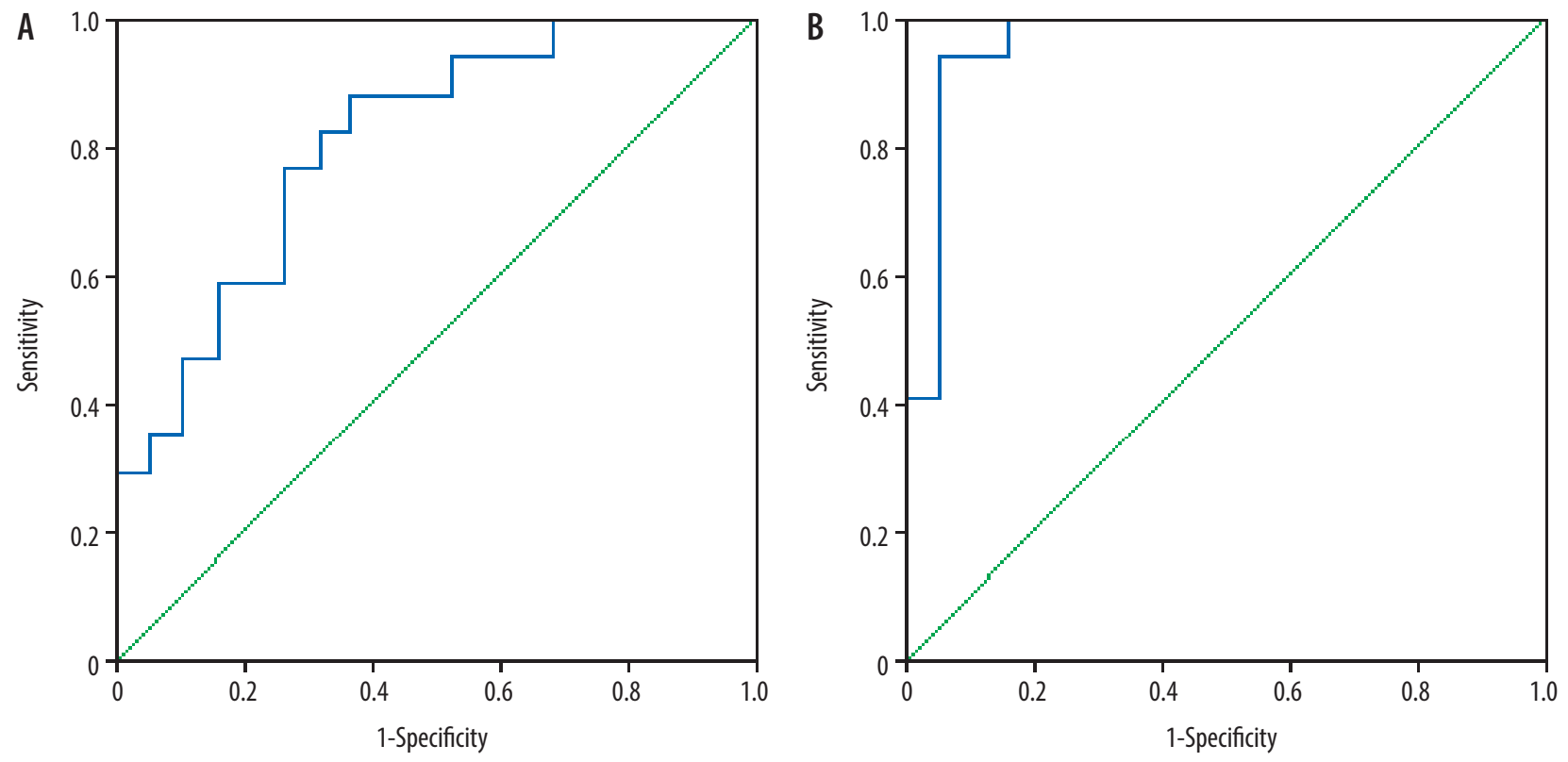

Figure 4. ROC curve of combined tumour blood flow (TBF) and apparent diffusion coefficient (ADC). A) Combined TBF and ADC of the tumoural region revealed AUC of 0.808 and accuracy of $72.7 \%$. B) Combined TBF and ADC of the peritumoural region revealed AUC of 0.96 and accuracy of $94.4 \%$

Second, this study was done using a 1.5 Tesla scanner. Further studies using a 3 tesla scanner are recommended to improve the results. Third, this study used ASL and ADC; further studies with application of diffusion tensor imaging [40-42], perfusion MR imaging [43-47], and proton MR spectroscopy [48-50] will improve the grading of gliomas. Fourth, there was no genetic correlation. Further studies with correlation of genetic studies and the molecular classification introduced by the 2016 WHO classification will help to better characterise diffuse gliomas.

\section{Conclusions}

We conclude that combined TBF and ADC of tumoural and peritumoural regions is a non-invasive method that improves the diagnostic power of discrimination between grade II and grade III gliomas.

\section{Conflict of interest}

The authors declare no conflict of interest.

\section{References}

1. Lin AL, DeAngelis LM. Reappraising the 2016 WHO classification for diffuse glioma. Neuro Oncol 2017; 19: 609-610.

2. Cimino PJ, Zager M, McFerrin L, et al. Multidimensional scaling of diffuse gliomas: application to the 2016 World Health Organization classification system with prognostically relevant molecular subtype discovery. Acta Neuropathol Commun 2017; 5: 39.

3. Sasaki H, Yoshida K. Treatment recommendations for adult patients with diffuse gliomas of grades II and III according to the new WHO classification in 2016. Neurol Med Chir (Tokyo) 2017; 57: 658-66.

4. Van Den Bent MJ, Smits M, Kros JM, Chang SM. Diffuse infiltrating oligodendroglioma and astrocytoma. J Clin Oncol 2017; 35: 23942401.

5. Delgado-López PD, Corrales-García EM, Martino J, Lastra-Aras E, Dueñas-Polo MT. Diffuse low-grade glioma: a review on the new molecular classification, natural history and current management strategies. Clin Transl Oncol 2017; 19: 931-944.

6. Van Den Bent MJ, Bromberg JE, Buckner J. Low-grade and anaplastic oligodendroglioma. Handb Clin Neurol 2016; 134: 361-380.
7. Tabouret E, Nguyen AT, Dehais C, et al. Prognostic impact of the 2016 WHO classification of diffuse gliomas in the French POLA cohort. Acta Neuropathol 2016; 132: 625-634.

8. Appin CL, Brat DJ. Biomarker-driven diagnosis of diffuse gliomas. Mol Aspects Med 2015; 45: 87-96.

9. Delfanti RL, Piccioni DE, Handwerker J, et al. Imaging correlates for the 2016 update on WHO classification of grade II/III gliomas: implications for IDH, 1p/19q and ATRX status. J Neurooncol 2017; 135: 601-609.

10. Arevalo-Perez J, Kebede AA, Peck KK, et al. Dynamic Contrast-Enhanced MRI in Low-Grade Versus Anaplastic Oligodendrogliomas. J Neuroimaging 2016; 26: 366-371.

11. Delgado AF, Delgado AF. Discrimination between glioma grades II and III using dynamic susceptibility perfusion MRI: a meta-analysis. AJNR Am J Neuroradiol 2017; 38: 1348-1355.

12. Falk A, Fahlström M, Rostrup E, et al. Discrimination between glioma grades II and III in suspected low-grade gliomas using dynamic contrast-enhanced and dynamic susceptibility contrast perfusion MR imaging: a histogram analysis approach. Neuroradiology 2014; 56: 1031-1038. 
13. Jia Z, Geng D, Liu Y, Chen X, Zhang J. Low-grade and anaplastic oligodendrogliomas: differences in tumour microvascular permeability evaluated with dynamic contrast-enhanced magnetic resonance imaging. J Clin Neurosci 2013; 20: 1110-1113.

14. Delgado AF, Fahlström M, Nilsson M, et al. Diffusion kurtosis imaging of gliomas grades II and III - a study of perilesional tumor infiltration, tumor grades and subtypes at clinical presentation. Radiol Oncol 2017; 51: 121-129.

15. Goebell E, Paustenbach S, Vaeterlein O, et al. Low-grade and anaplastic gliomas: differences in architecture evaluated with diffusion-tensor MR imaging. Radiology 2006; 239: 217-222.

16. Xing Z, Yang X, She D, Lin Y, Zhang Y, Cao D. Assessment of IDH mutational status in world health organization grade II and III astrocytomas using DWI and DSC-PWI combined with conventional MR imaging. AJNR Am J Neuroradiol 2017; 38: 1138-1144.

17. Leu K, Ott GA, Lai A, et al. Perfusion and diffusion MRI signatures in histologic and genetic subtypes of WHO grade II-III diffuse gliomas. J Neurooncol 2017; 134: 177-188.

18. Razek AAKA, El-Serougy L, Abdelsalam M, Gaballa G, Talaat M. Differentiation of residual/recurrent gliomas from postradiation necrosis with arterial spin labeling and diffusion tensor magnetic resonance imaging-derived metrics. Neuroradiology 2018; 60: 169-177.

19. Zeng Q, Jiang B, Shi F, Ling C, Dong F, Zhang J. 3D Pseudocontinuous arterial spin-labeling MR imaging in the preoperative evaluation of gliomas. AJNR Am J Neuroradiol 2017; 38: 1876-1883.

20. Abdel Razek AAK, Nada N. Arterial spin labeling perfusion-weighted MR imaging: correlation of tumor blood flow with pathological degree of tumor differentiation, clinical stage and nodal metastasis of head and neck squamous cell carcinoma. Eur Arch Otorhinolaryngol 2018; 275: 1301-1307.

21. Brendle C, Hempel JM, Schittenhelm J, et al. Glioma grading and determination of IDH mutation status and ATRX loss by DCE and ASL perfusion. Clin Neuroradiol 2018; 28: 421-428.

22. Kong L, Chen H, Yang Y, Chen L. A meta-analysis of arterial spin labelling perfusion values for the prediction of glioma grade. Clin Radiol 2017; 72: 255-261.

23. Furtner J, Schöpf V, Schewzow K, et al. Arterial spin-labeling assessment of normalized vascular intratumoral signal intensity as a predictor of histologic grade of astrocytic neoplasms. AJNR Am J Neuroradiol 2014; 35: 482-489.

24. Yang S, Zhao B, Wang G, et al. Improving the Grading Accuracy of Astrocytic Neoplasms Noninvasively by Combining Timing Information with Cerebral Blood Flow: A Multi-TI Arterial Spin-Labeling MR Imaging Study. AJNR Am J Neuroradiol 2016; 37: 2209 2216.

25. Abdel Razek AAK, Talaat M, El-Serougy L, Gaballa G, Abdelsalam M. Clinical applications of arterial spin labeling in brain tumors. J Comput Assist Tomogr 2019; 43: 525-532.

26. Abdel Razek AAK, Talaat M, El-Serougy L, Abdelsalam M, Gaballa G. Differentiating glioblastomas from solitary brain metastases using arterial spin labeling perfusion - and diffusion tensor imaging-derived metrics. World Neurosurg 2019; 127: e593-e598.

27. Abdel Razek AAK, El-Serougy L, Abdelsalam M, Gaballa G, Talaat M. Differentiation of primary central nervous system lymphoma from glioblastoma: quantitative analysis using arterial spin labe- ling and diffusion tensor imaging. World Neurosurg 2019; 123 : e303-e309.

28. Abdel Razek AAK. Arterial spin labelling and diffusion-weighted magnetic resonance imaging in differentiation of recurrent head and neck cancer from post-radiation changes. J Laryngol Otol 2018; 132 : 923-928.

29. Zeng Q, Dong F, Shi F, Chenhan L, Jiang B, Zhang J. Apparent diffusion coefficient maps obtained from high $\mathrm{b}$ value diffusion-weighted imaging in the preoperative evaluation of gliomas at 3T: comparison with standard b value diffusion-weighted imaging. Eur Radiol 2017; 27: 5309-5315.

30. Han H, Han C, Wu X, et al. Preoperative grading of supratentorial nonenhancing gliomas by high $\mathrm{b}$-value diffusion-weighted $3 \mathrm{~T}$ magnetic resonance imaging. J Neuroonco 2017; 133: 147-154.

31. Maximov II, Tonoyan AS, Pronin IN. Differentiation of glioma malignancy grade using diffusion MRI. Phys Med 2017; 40: 24-32.

32. El-Serougy L, Abdel Razek AA, Ezzat A, Eldawoody H, El-Morsy A. Assessment of diffusion tensor imaging metrics in differentiating low-grade from high-grade gliomas. Neuroradiol J 2016; 29: 400-407.

33. Hu YC, Yan LF, Sun Q, et al. Comparison between ultra-high and conventional mono b-value DWI for preoperative glioma grading. Oncotarget 2017; 8: 37884-37895.

34. Abdel Razek AA, Kamal E. Nasopharyngeal carcinoma: correlation of apparent diffusion coefficient value with prognostic parameters. Radiol Med 2013; 118: 534-539.

35. Abdel Razek AA, Elkhamary S, Al-Mesfer S, Alkatan HM. Correlation of apparent diffusion coefficient at $3 \mathrm{~T}$ with prognostic parameters of retinoblastoma. AJNR Am J Neuroradiol 2012; 33: 944-948.

36. Abdel Razek A, Mossad A, Ghonim M. Role of diffusion-weighted MR imaging in assessing malignant versus benign skull-base lesions. Radiol Med 2011; 116: 125-132.

37. Abdel Razek AA, Soliman N, Elashery R. Apparent diffusion coefficient values of mediastinal masses in children. Eur J Radiol 2012; 81: 1311-1314.

38. Surov A, Nagata S, Razek AA, Tirumani SH, Wienke A, Kahn T. Comparison of ADC values in different malignancies of the skeletal musculature: a multicentric analysis. Skeletal Radiol 2015; 44: 995-1000

39. Abdel Razek AA, El-Hadidy EM, Moawad ME, El-Metwaly N, El-Hamid El-Said AA. Assessment of lacrimal glands in thyroid eye disease with Diffusion-weighted MR imaging. Polish J Radiol 2019; 84: e142-e146.

40. Razek AAKA. Diffusion tensor imaging in differentiation of residual head and neck squamous cell carcinoma from post-radiation changes. Magn Reson Imaging 2018; 54: 84-89.

41. Khalek Abdel Razek AA. Characterization of salivary gland tumours with diffusion tensor imaging. Dentomaxillofac Radiol 2018; 47: 20170343

42. Abdel Razek AAK. Routine and advanced diffusion imaging modules of the salivary glands. Neuroimaging Clin N Am 2018; 28: $245-$ 254.

43. Abdel Razek AA, Samir S, Ashmalla GA. Characterization of Parotid Tumors With Dynamic Susceptibility Contrast Perfusion-Weighted Magnetic Resonance Imaging and Diffusion-Weighted Mr Imaging. J Comput Assist Tomogr 2017;41:131-6. 
44. Abdel Razek AA, Gaballa G. Role of perfusion magnetic resonance imaging in cervical lymphadenopathy. J Comput Assist Tomogr 2011; 35: 21-25.

45. Abdel Razek AA, Gaballa G, Ashamalla G, Alashry MS, Nada N. Dynamic susceptibility contrast perfusion-weighted magnetic resonance imaging and diffusion-weighted magnetic resonance imaging in differentiating recurrent head and neck cancer from postradiation changes. J Comput Assist Tomogr 2015; 39: 849-854.

46. Razek AAKA. Multi-parametric MR imaging using pseudo-continuous arterial-spin labeling and diffusion-weighted MR imaging in differentiating subtypes of parotid tumors. Magn Reson Imaging 2019; 63: 55-59.

47. Razek AA, Abdalla A, Gaber NA, et al. Proton MR Spectroscopy of the brain in children with neuronopathic Gaucher's disease. Eur Radiol 2013; 23: 3005-3011.
48. Razek AA, Nada N. Correlation of choline/creatine and apparent diffusion coefficient values with the prognostic parameters of head and neck squamous cell carcinoma. NMR Biomed 2016; 29: 483-489.

49. Razek AA, Abdalla A, Ezzat A, Megahed A, Barakat T. Minimal hepatic encephalopathy in children with liver cirrhosis: diffusion-weighted MR imaging and proton MR spectroscopy of the brain. Neuroradiology 2014; 56: 885-891.

50. El-Mewafy MZ, Abdel Razek AAAK, El-Eshmawy M, El-Eshmawy MM, El-Eneen NRA, El-Biaomy AAB. MR spectroscopy of the frontal region in patients with metabolic syndrome: Correlation with anthropometric measurement. Pol J Radiol 2018; 83: e215-e219. 